\title{
Linoleate metabolism in multiple sclerosis
}

\author{
J. Belin, N. Pettet, A. D. SMith, R. H. S. ThOMPSON, AND K. J. Zilkha \\ From the Courtauld Institute of Biochemistry, The Middlesex Hospital Medical School, London, \\ and The National Hospital, Queen Square, London
}

SUMMARY (1) The levels of oleate and linoleate in the serum have been measured in 14 patients with multiple sclerosis (MS) and in 14 healthy subjects before, during and after a five day period when the normal diet was supplemented with linoleate. (2) In confirmation of earlier work the mean percentage of linoleate in the serum lipids of the MS patients was significantly lower $(\mathrm{P}<0.01)$ than in the control subjects in the pre-supplementation period. The mean percentage of oleate showed an increase $(P<0.005)$ in the patients as compared with the controls while on their normal diets. (3) The period of linoleate feeding produced a considerable rise in the percentage of linoleate together with a fall in the percentage of oleate in both the controls and the MS patients. (4) When large amounts of linoleate, as present in sunflower seed oil, are ingested we have been unable to obtain evidence of a defect in absorption from the intestinal lumen.

It has been shown by several groups of workers that in multiple sclerosis there are changes in the relative proportions of saturated and unsaturated fatty acids in the brain lipids (Baker, Thompson, and Zilkha, 1963; Gerstl, Tavaststjerna, Hayman, Eng, and Smith, 1965; Cumings, Shortman, and Skrbic, 1965; Arnetoli, Pazzagli, and Amaducci, 1969), and also that there is a lowering of the level of linoleate in the serum (Thompson, 1966). More recently, Gul, Smith, Thompson, Payling Wright, and Zilkha (1970) have found that the relative level of linoleate, expressed as a percentage of the five main fatty acids, is also reduced both in the platelets and in the red blood cells of patients with multiple sclerosis. It is relevant also to note that Clausen and Møller (1967) have reported that rats bred and raised on a diet deficient in polyunsaturated fatty acid show an increased susceptibility to the development of allergic encephalomyelitis after the injection of a brain antigen.

In view, therefore, of this growing evidence in multiple sclerosis of an apparently widespread abnormality in the handling of certain fatty acids by the body, and since the view has been put forward that the high prevalence of multiple sclerosis in certain parts of the world may be related to a dietary deficiency of unsaturated fatty acids (Swank, 1950; Sinclair, 1956; Allison, 1963), it seemed important to determine whether any difference could be revealed between healthy subjects and patients with multiple sclerosis in their ability to incorporate orally administered linoleate into the various types of plasma lipid.

It is well known that the feeding of diets with a high content of linoleate causes an increase of linoleate in the serum lipid fractions together with a decrease of oleate and sometimes of palmitate, palmitoleate, and stearate as well. In comparatively few cases have these changes been studied over a short time interval (Irwin and Wiese, 1961; Gunning, Michaels, Neumann, Splitter, and Kinsell, 1963; Nichaman, Sweeley, and Olson, 1967), although indications exist that an increase of linoleate in the $\beta$-lipoproteins can be observed within hours after a single dose of corn oil (Farquhar and Ahrens, 1963).

In a series of healthy control subjects and of patients with multiple sclerosis we have therefore measured the fatty acid patterns in serum, drawn after an overnight fast, both before and during a five day period when the normal diet was supplemented with linoleate, and during the two days after the cessation of these supplements. We have also studied the relative proportions of fatty acids in the separated triglyceride, cholesteryl ester, and phospholipid fractions of the serum samples.

\section{EXPERIMENTAL}

DESIGN OF EXPERIMENTS Blood samples were taken from the antecubital vein after an overnight fast on three days while the subject was on his or her normal diet, during the week before the start of the linoleate supplements. 
The supplements were then added to the normal diet, and were continued for a five day period; blood samples were taken, each after an overnight fast, throughout this period, and a further two samples were taken on the two consecutive days after cessation of the linoleate supplements.

Two doses of the linoleate supplements were taken each day, morning and evening. Each dose consisted of $50 \mathrm{ml}$. of a sunflower seed oil emulsion containing $14.3 \mathrm{~g}$ linoleic acid, which was prepared for use in a controlled therapeutic trial which is being undertaken by one of us (K.J.Z.) together with Dr. H. Millar and Dr. M. Langman. The emulsion was made up as follows:

$50 \mathrm{ml}$. sunflower seed oil $\quad 0.025 \mathrm{~g}$ Orange $\mathrm{G}$ dye

$1.4 \mathrm{~g}$ methyl cellulose $20 \quad 2 \mathrm{ml}$. benzoic acid solution

$0.18 \mathrm{~g}$ citric acid

$0.02 \mathrm{~g}$ saccharin sodium $\quad 0.4 \mathrm{ml}$. orange flavour

Water to $100 \mathrm{ml}$.

CONTROLS AND PATIENTS Fourteen members of the staff of The Middlesex Hospital Medical School acted as healthy controls for the experiment; their ages ranged from 19 to 59 years (mean $=36.3$ years), and there were eight males and six females.

The 14 patients with multiple sclerosis consisted of seven males and seven females, whose ages ranged from 20 to 53 years $($ mean $=36.6$ years), and were hospitalized throughout the 10 day experimental period, 13 of them in the National Hospital, Queen Square, London, and one in King's College Hospital, London. All the patients except one were fully ambulant, and were on a normal diet. The one bedridden patient with severe paraplegia and ataxia sat in a chair for one hour each day. He also took a normal hospital diet. Two of the patients were receiving $\mathrm{ACTH}$ at the time of the investigation, and one was on imipramine. The disease was in an active stage in all patients, and nine of them showed recent deterioration during the previous month.

MATERIALS Methanol was B.D.H. Analar grade. Other solvents were May and Baker reagent grade. Hexane, chloroform, benzene, and methanol were redistilled before use. One per cent $(w / w)$ ethanol was added to the chloroform after distillation. Nitrogen was oxygen-free. Silicic acid was washed and activated as described by Rouser, O'Brien, and Heller (1961).

EXTRACTION OF LIPIDS Lipids were extracted by the method of Folch, Lees, and Sloane Stanley (1957). For the analysis of total fatty acids $0.5 \mathrm{ml}$. serum samples were taken, and for the fractionation experiments between 1.5 and $2.5 \mathrm{ml}$. For the analysis of total fatty acids an internal standard (C 21:0 methyl ester) was added to the serum sample before extraction to permit calculation of actual amounts of fatty acid present in $\mathrm{mg} / \mathrm{ml}$.

FRACTIONATION OF LIPIDS For the fractionation experiments the total lipid extract, after being taken to dryness, was loaded onto a silicic acid column $(1 \mathrm{~cm}$ diameter, $2.5 \mathrm{~g}$ ) and fractionated, as described by Blomstrand, Gürtler, and Werner (1965), to obtain cholesteryl esters, triglycerides, free fatty acids, and phospholipids. The free fatty acids were present in quantities too small for accurate measurement, and were discarded.

SAPONIFICATION After removal of solvent under nitrogen the total lipid extract (for total fatty acids) or the column fractions (for fatty acids of lipid classes) were incubated with $2 \mathrm{ml}$. ethanolic $\mathrm{KOH}(6 \mathrm{ml} .33 \%$ aqueous $\mathrm{KOH}$ and $94 \mathrm{ml}$. ethanol containing $4 \mathrm{mg}$ hydroquinone) for $1 \mathrm{hr}$ at $80^{\circ} ; 2 \mathrm{ml}$. water were then added and the incubation continued at $80^{\circ}$ for a further $10 \mathrm{~min}$. After cooling, 1 drop of thymol blue was added, and the solution extracted twice with $3 \mathrm{ml}$. hexane. These hexane extracts were discarded, the alkaline lower layer acidified with $1 \mathrm{~N} \mathrm{HCl}$, and fatty acids extracted into $3 \mathrm{ml}$. hexane. The fatty acid extract was then taken to dryness under nitrogen, dissolved in $0.2 \mathrm{ml}$. hexane, and methylated using diazomethane.

GAS CHROMATOGRAPHY The methyl esters were run on $20 \%$ diethylene glycol succinate columns using a PerkinElmer F11 gas chromatograph.

\section{RESULTS}

Table 1 summarizes the results of the measurements of the levels of oleate $(18: 1)$ and linoleate $(18: 2)$ expressed as percentages of the total fatty acids, i $\mathrm{i}$. the unfractionated fasting serum samples obtaine웅 before, during, and after administration of the

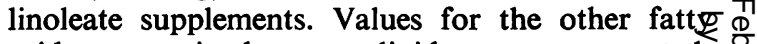
acids present in the serum lipids are not presentedo as, with the exception of palmitate, the linoleate feeding produced no significant changes in their levels, while for palmitate (as well as for the othep fatty acids measured) at no time was there a signi- :ficant difference between the controls and the patients.

In the case of both the healthy controls and the multiple sclerosis patients the percentages of oleate and linoleate remained remarkably constant in the fasting specimens drawn during the period while they were living on their various normal diets before the linoleate supplementation (see Fig.); the individual variations within each of the two groups were also small. The mean levels for the males and for the females throughout this period did not differ significantly; nor was there any correlation with the age of the subject.

In confirmation of our earlier work, the mean level of linoleate for the multiple sclerosis patients $(29.7 \%)$ was significantly lower $(P<0.01)$ than that of the control subjects $(33.7 \%)$ during the presupplementation period. It will be seen that this was accompanied by an inverse difference in the percentages of oleate, resulting in the ratio 18:2/18:1 being decreased from 1.35 for the controls to 1.05 for the patients $(P<0.001)$.

In the controls the five day period of linoleate 
TABLE 1

PERCENTAGE CONTENT OF OLEATE (18:1) AND LINOLEATE (18:2) IN SERUM LIPIDS OF CONTROLS AND OF MS PATIENTS BEFORE, DURING, AND AFTER FIVE DAY PERIOD OF SUPPLEMENTATION OF NORMAL DIETS WITH SUNFLOWER SEED OIL EMULSION

\begin{tabular}{|c|c|c|c|c|c|c|c|c|c|}
\hline & & \multirow{2}{*}{$\begin{array}{l}\text { Pre-oil } \\
\text { (mean) }\end{array}$} & \multicolumn{5}{|c|}{ During supplementation with oil } & \multicolumn{2}{|c|}{ Post-oil } \\
\hline & & & 1 & 2 & 3 & 4 & 5 & $l$ & 2 \\
\hline $\begin{array}{c}18: 1 \\
\% \pm \text { SEM }\end{array}$ & $\begin{array}{l}\text { Control } \\
\text { MS }\end{array}$ & $\begin{array}{c}25.4 \\
\pm 0.5 \\
(14) \\
28.9 \\
\pm 0.6 \\
(14)\end{array}$ & $\begin{array}{c}21.7 \\
\pm \quad 0.8 \\
(5) \\
25.2 \\
\pm \quad 0.9 \\
(4)\end{array}$ & $\begin{array}{c}21.6 \\
\pm 0.6 \\
(7) \\
24.4 \\
+\quad 1.3 \\
(4)\end{array}$ & $\begin{array}{c}19.8 \\
\pm \quad 0.6 \\
(10) \\
22.6 \\
\pm \quad 0.5 \\
(13)\end{array}$ & $\begin{array}{c}20.2 \\
\pm 0.6 \\
(10) \\
21.8 \\
\pm 0.6 \\
(12)\end{array}$ & $\begin{array}{c}20.8 \\
\pm \quad 1.3 \\
(7) \\
21.5 \\
\pm \quad 0.6 \\
(14)\end{array}$ & $\begin{array}{c}21.8 \\
+\quad 0.6 \\
(10) \\
24.0 \\
+0.6 \\
(11)\end{array}$ & $\begin{array}{r}22.8 \\
+\quad 0.7 \\
(13) \\
25.5 \\
\pm 0.5 \\
(14)\end{array}$ \\
\hline $\begin{array}{c}18: 2 \\
\% \pm \text { SEM }\end{array}$ & $\begin{array}{l}\text { Control } \\
\text { MS }\end{array}$ & $\begin{array}{r}33.7 \\
+\quad 1.0 \\
(14) \\
29.7 \\
+\quad 0.8 \\
(14)\end{array}$ & $\begin{array}{c}40.0 \\
+\quad 1.8 \\
(5) \\
36.0 \\
\pm \quad 1.3 \\
(4)\end{array}$ & $\begin{array}{c}41 \cdot 4 \\
\pm \quad 1 \cdot 1 \\
(7) \\
37 \cdot 7 \\
\pm \quad 1 \cdot 5 \\
(4)\end{array}$ & $\begin{array}{c}42.5 \\
+\quad 1.3 \\
(10) \\
39.9 \\
\pm 0.9 \\
(13)\end{array}$ & $\begin{array}{c}42.5 \\
+\quad 1.4 \\
(10) \\
41.1 \\
+\quad 0.9 \\
(12)\end{array}$ & $\begin{array}{c}42.5 \\
\pm \quad 1.9 \\
(7) \\
41.2 \\
\pm \quad 1.2 \\
(14)\end{array}$ & $\begin{array}{c}39.5 \\
+\quad 1.3 \\
(10) \\
36.7 \\
+\quad 0.8 \\
(11)\end{array}$ & $\begin{array}{c}38.1 \\
+\quad 1.4 \\
(13) \\
34 \cdot 1 \\
+\quad 0.9 \\
(14)\end{array}$ \\
\hline $18: 2 *$ & Control & $\begin{aligned} & 1.35 \\
&+ 0.06 \\
&(14)\end{aligned}$ & $\begin{aligned} & 1.87 \\
&+ 0.14 \\
&(5)\end{aligned}$ & $\begin{aligned} & 1.98 \\
&+\quad 0.10 \\
&(7)\end{aligned}$ & $\begin{aligned} & 2 \cdot 19 \\
&+\quad 0.13 \\
&(10)\end{aligned}$ & $\begin{aligned} 2.14 \\
+\quad 0.12 \\
(10)\end{aligned}$ & $\begin{aligned} & 2 \cdot 12 \\
&+ 0 \cdot 20 \\
&(7)\end{aligned}$ & $\begin{array}{l}1.84 \\
+\quad 0.10 \\
(10)\end{array}$ & $\begin{array}{l}1.72 \\
=\quad 0.14 \\
(13)\end{array}$ \\
\hline $\begin{array}{c}\overline{18: 1} \\
\% \pm \text { SEM }\end{array}$ & MS & $\begin{aligned} & 1.05 \\
&+ 0.05 \\
&(14)\end{aligned}$ & $\begin{aligned} 1 \cdot 44 \\
+0.10 \\
(4)\end{aligned}$ & $\begin{array}{l}1.56 \\
\pm 0.14 \\
(4)\end{array}$ & $\begin{aligned} 1.78 \\
\pm \quad 0.08 \\
(13)\end{aligned}$ & $\begin{array}{r}1.91 \\
\pm 0.09 \\
(12)\end{array}$ & $\begin{array}{r}1.95 \\
+\quad 0.09 \\
(14)\end{array}$ & $\begin{array}{r}1.54 \\
+\quad 0.06 \\
(11)\end{array}$ & $\begin{aligned} & 1.35 \\
&+ 0.6 \\
&(14)\end{aligned}$ \\
\hline
\end{tabular}

*Calculated as ratio of fatty acid levels in $\mathrm{mg} / \mathrm{ml}$. serum.

Numbers of subjects in parentheses.

Controls $v$ MS in pre-oil period

Controls v MS after four days' supplementation

Controls v MS after five days' supplementation

Controls $v$ MS one day after end of supplementation

Controls v MS two days after end of supplementation

$\begin{array}{ccc}\begin{array}{c}\% 18.1 \\ \mathrm{P}<0.005\end{array} & \mathrm{P}<0.01 & 18: 2 / 18: 1 \\ \mathrm{NS} & \mathrm{NS} & \mathrm{P}<0.001 \\ \mathrm{NS} & \mathrm{NS} & \mathrm{NS} \\ \mathrm{P}<0.02 & \mathrm{NS} & \mathrm{P}<0.02 \\ \mathrm{P}<0.01 & \mathrm{P}<0.05 & \mathrm{P}<0.02\end{array}$

feeding produced a considerable and consistent rise in the percentage of linoleate, accompanied by a corresponding fall in the percentages of oleate (see Fig.) and of palmitate. These changes were evident after only one day's linoleate supplementation, and, over the five days studied, appeared to level off to maximum changes by three to four days. Samples taken one and two days after the end of

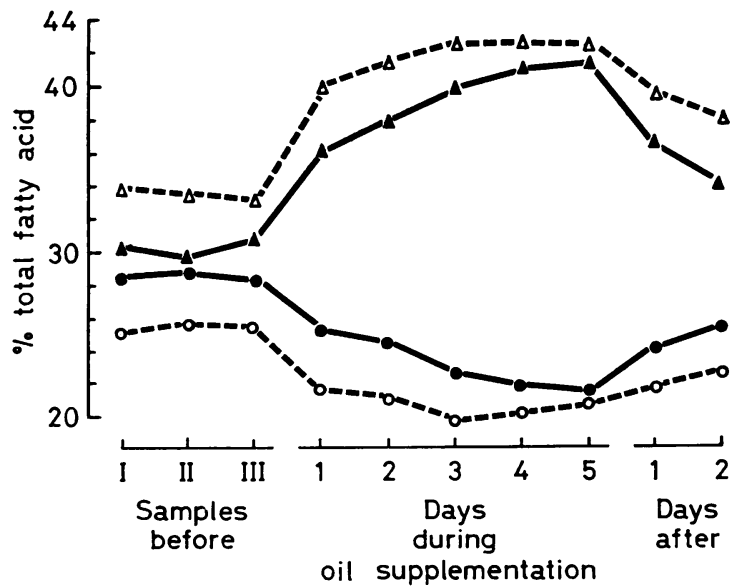

the linoleate supplementation showed a progressive partial return to the pre-supplementation levels for each of these three acids.

Table 1 also shows that, although the multiple sclerosis patients had lower pre-linoleate levels of $18: 2$, these also rose strikingly during the period of dietary supplementation. Indeed, as will be seen from Table 2, the changes in the percentages of both

FIG. Mean levels of oleate and linoleate in the serum of controls and MS patients, expressed as percent of the total fatty acids before, during, and after the supplementation of the diet with sunflower seed oil emulsion. $\bigcirc=$ oleate in controls, oleate in MS patients, $\triangle=$ linoleate in controls. $\Delta=$ linoleate in MS patients. 
TABLE 2

CHANGES FROM MEAN 'PRE-OIL' LEVEL IN PERCENTAGES OF OLEATE (18:1) AND LINOLEATE (18:2) IN SERUM LIPIDS PRODUCED BY FEEDING SUNFLOWER SEED OIL EMULSION

\begin{tabular}{llccccc}
\hline $\begin{array}{c}\text { Days on sunflower seed } \\
\text { oil supplements }\end{array}$ & 1 & 2 & 3 & 4 & 5 \\
\hline $18: 1$ & Controls & -3.8 & -4.3 & -5.6 & -5.2 & $-4 \cdot 6$ \\
& MS & -3.6 & -4.3 & -6.2 & -7.0 & -7.4 \\
$18: 2$ & Controls & +6.3 & +7.7 & +8.8 & +8.8 & +8.8 \\
& MS & +6.3 & +8.0 & +10.2 & +11.5 & +11.5 \\
\hline
\end{tabular}

18:2 and 18:1 in the multiple sclerosis patients produced by the linoleate feeding were even greater for days 2 to 5 of the feeding period than those produced in the controls, so that by day 4 of the feeding period no statistically significant difference existed between the control and multiple sclerosis groups in the percentages of either $18: 2$ or $18: 1$ (Table 1). On the other hand, in the post-feeding period the level of both 18:2 and 18:1 in the multiple sclerosis sera appeared to revert rather more quickly towards the pre-feeding levels than did the levels in the control sera, so that by the second day after the end of the feeding period a statistically significant difference had reappeared between the two groups of subjects.

Fatty acid measurements were carried out on the separated triglyceride, cholesteryl ester, and phospholipid fractions of the serum samples from 10 controls and 10 patients. In each of these fractions obtained from the pre-supplementation serum samples-that is, from patients existing on their normal diets - the percentage of 18:2 was lower, and of 18:1 higher, in the multiple sclerosis patients than in the controls. The indications from this preliminary work are that there may be significant differences in the extent of the changes in fatty acid percentages in the different fractions in the two groups of subjects as a result of the supplementation. Further work is in progress to elucidate this possibility.

\section{DISCUSSION}

In addition to confirming our earlier findings of low levels of linoleate in the serum of patients with multiple sclerosis the observations described above show also that the percentage of oleate is increased in these patients, so that the $18: 2 / 18: 1$ ratio is strikingly changed (about $30 \%$ ). We have also shown that on the linoleate intake provided by the normal diets taken by our control subjects and by our multiple sclerosis patients there appears, in the case of the patients, to be a failure to incorporate linoleate into each of the three lipid fractions studied.
It is moreover clear that changes in the levels of 18:1 are prominent, and indeed, in the 14 cases under study, statistically more significant between patients and controls than the changes in linoleate. Previous work has shown, and is confirmed by the present results, that an inverse relationship seems to exist between oleate and linoleate. The differences in oleate levels may therefore be a reflection of the differences in linoleate. However, we feel that it may be unwise, in speculating on the role which fatty acids may play in multiple sclerosis, to pay exclusive attention to one acid, or 'family' of acidsthat is, linoleate and its metabolic derivatives. For example, where changes in the proportions of fatty acids in the brain have been demonstrated in multiple sclerosis, it is noteworthy that in three laboratories (Baker et al., 1963; Cumings et al., 1965; Arnetoli et al., 1969) the most prominent change observed is in 18:1 (oleate or its isomers), which is decreased in multiple sclerosis. Moreover, it is difficult using existing biochemical knowledge to suggest any connection between low serum linoleate and low brain oleate, and it may well be that the connection, if one exists, will be revealed only with further advances in fundamental knowl edge.

The results reported have shown, generally, that when large amounts of linoleate are available fop $\mathbb{D}$ absorption from the intestinal lumen, they bring 0 about a marked rise in the level of linoleate in theg serum (Table 2) which, we presume, indicates that considerable quantities are being absorbed both in the controls and in the patients. On present evidence, therefore, we are unable to account for the low serum linoleate levels found in multiple sclerosis patients on their normal unsupplemented diets. Various possibilities could, however, be suggested: for example it is possible, in terms of the known pathways for the metabolism of triglycerides, cholesteryl esters, and phospholipids, that a different metabolic pathway or pathways may come into operation when the amount of available linoleate is increased dramatically, as in the supplements which we have used in this work, and that in multiple sclerosis these pathways may be functioning normally, whereas in the absence of these large amounts of available linoleate an alternative metabolic pathway is in operation, which is defective in multiple sclerosis, the defect revealing itself in the slight but consistent lowering in the percentage of 18:2 incorporated into the different lipids. Alternatively, it may be that these large supplements of linoleate are sufficient to correct an abnormally high rate of utilization or loss of linoleate in patients on their normal diet. It is our hope that further experimental work may throw some light on the mechanisms, and 
their control, concerned in the incorporation of linoleate into these lipid fractions in multiple sclerosis. Meanwhile, however, our findings would appear to suggest that, since linoleate feeding can cause significant elevations of the low linoleate levels found in multiple sclerosis, a therapeutic trial of linoleate supplementation, such as is at present being carried out, is justified.

We wish to acknowledge with gratitude the help received from our clinical colleagues who allowed us access to cases under their care and who supervised the administration of the emulsion to the patients while in hospital. Our thanks are also due to those colleagues who acted as control subjects in this investigation, and to the Medical Research Council and the Multiple Sclerosis Society for grants in support of their work.

\section{REFERENCES}

Allison, R. S. (1963). Some neurological aspects of medical geography. Proc. roy. Soc. Med., 56, 71-76.

Arnetoli, G., Pazzagli, A., and Amaducci, L. (1969). Fatty acid and aldehyde changes in choline- and ethanolaminecontaining phospholipids in the white matter of multiple sclerosis brains. J. Neurochem., 16, 461-463.

Baker, R. W. R., Thompson, R. H. S., and Zilkha, K. J. (1963). Fatty-acid composition of brain lecithins in multiple sclerosis. Lancet, 1, 26-27.

Blomstrand, R., Gurtler, J., and Werner, B. (1965). Intestinal absorption and esterification of ${ }^{14} \mathrm{C}$-labelled fatty acids in man. J. clin. Invest., 44, 1766-1777.

Clausen, J., and Møller, J. (1967). Allergic encephalomyelitis induced by brain antigen after deficiency in polyunsaturated fatty acids during myelination. Is multiple sclerosis a nutritive disorder? Acta neurol. scand., 43, 375-388.
Cumings, J. N., Shortman, R. C., and Skrbic, T. (1965). Lipid studies in the blood and brain in multiple sclerosis and motor neurone disease. J. clin. Path., 18, 641-644.

Farquhar, J. W., and Ahrens, E. H., Jr. (1963). Effects of dietary fats on human erythrocyte fatty acid patterns. J. clin. Invest., 42, 675-685.

Folch, J., Lees, M., and Sloane Stanley, G. H. (1957). A simple method for the isolation and purification of total lipides from animal tissues. J. biol. Chem., 226, 497-509.

Gerstl, B., Tavaststjerna, M. C., Hayman, R. B., Eng, L. F., and Smith, J. K. (1965). Alterations in myelin fatty acids and plasmalogens in multiple sclerosis. Ann. N.Y. Acad. Sci., 122, 405-416.

Gul, S., Smith, A. D., Thompson, R. H. S., Payling Wright, H., and Zilkha, K. J. (1970). The fatty acid composition of phospholipids from platelets and erythrocytes in multiple sclerosis. J. Neurol. Neurosurg. Psychiat., 33, 506-510.

Gunning, B., Michaels, G., Neumann, L., Splitter, S., and Kinsell, L. (1963). Effects of a diet high in polyunsaturated fat on the plasma lipids of normal young females. J. Nutr., 79, 85-92.

Irwin, M. I., and Wiese, H. F. (1961). Variations in linoleic acid content of dietary fat in relation to metabolism of fat, nitrogen and minerals, and to changes in blood lipids. J. Nutr., 74, 217-225.

Nichaman, M. Z., Sweeley, C. C., and Olson, R. E. (1967). Plasma fatty acids in normolipemic and hyperlipemic subjects during fasting and after linoleate feeding. Amer. J. clin. Nutr., 20, 1057-1069.

Rouser, G., O'Brien, J., and Heller, D. (1961). The separation of phosphatidyl ethanolamine and phosphatidyl serine by column chromatography. J. Amer. Oil Chem. Soc., 38, 14-19.

Sinclair, H. M. (1956). Deficiency of essential fatty acids and atherosclerosis, etcetera. Lancet, 1, 381-383.

Swank, R. L. (1950). Multiple sclerosis: a correlation of its incidence with dietary fat. Amer. J. med. Sci., 220, 421-430.

Thompson, R. H. S. (1966). A biochemical approach to the problem of multiple sclerosis. Proc. roy. Soc. Med., 59, 269-276. 